Article

\title{
Changes in Bond Strength and Topography for Y-TZP Etched with Hydrofluoric Acid Depending on Concentration and Temperature Conditions
}

\author{
Hyo-Eun Kim ${ }^{\dagger}$, Myung-Jin Lim ${ }^{\dagger}$, Mi-Kyung Yu and Kwang-Won Lee * \\ Department of Conservative Dentistry, Jeonbuk National University School of Dentistry 567 Baekje-daero, \\ Jeonju 54896, Korea; hwa3106@naver.com (H.-E.K.); occasio00@naver.com (M.-J.L.); \\ mkyou102@jbnu.ac.kr (M.-K.Y.) \\ * Correspondence: lkw@jbnu.ac.kr; Tel.: +82-63-250-2016; Fax: +82-63-250-2129 \\ + These authors contributed equally to this work.
}

Received: 21 September 2020; Accepted: 26 October 2020; Published: 28 October 2020

\begin{abstract}
Background and objectives: This study aimed to investigate the change in bond strength between resin cement and tetragonal zirconia polycrystalline stabilized with 3 to $8 \mathrm{~mol} \%$ yttrium oxide (Y-TZP) and observe the topographical change of the Y-TZP surface when etched with hydrofluoric acid (HF) solution under different concentration and temperature conditions. Materials and Methods: Non-etched sintered Y-TZP specimens under two different temperature conditions (room temperature and $70-80{ }^{\circ} \mathrm{C}$, respectively), were used as a control, while experimental groups were etched with $5 \%, 10 \%, 20 \%$, and $40 \%$ HF solutions for $10 \mathrm{~min}$. After zirconia primer and MDP-containing resin cement were applied to the Y-TZP surface, the shear bond strength (SBS) of each experimental group was measured. Results: Under room temperature conditions, the highest SBS value was measured in the $40 \% \mathrm{HF}$ etching group, representing a significant deviation from the other groups $(p<0.05)$. In the $70-80{ }^{\circ} \mathrm{C}$ tests, the $40 \% \mathrm{HF}$ etching group also had the highest SBS value, but there was no significant difference when compared to the $20 \%$ HF etching group $(p>0.05)$. From SEM and AFM observations, the HF solution increasingly dissolved the Y-TZP surface grain structure as the concentration and application temperature rose, resulting in high surface roughness and irregularities. Conclusions: Pretreating with either $20 \% \mathrm{HF}$ solution at $70-80{ }^{\circ} \mathrm{C}$ or $40 \% \mathrm{HF}$ solution at room temperature and $70-80^{\circ} \mathrm{C}$ effectively acid etched the Y-TZP surface, resulting in more surface roughness and irregularities. Accounting for the concentration and temperature conditions of the HF solution, using $40 \% \mathrm{HF}$ solution at room temperature will result in improvements in adhesion between resin cement and Y-TZP.
\end{abstract}

Keywords: hydrofluoric acid; hot etching; shear bond strength; surface roughness; Y-TZP

\section{Introduction}

Zirconia restorations have superior mechanical properties compared with other ceramic systems used in dentistry [1,2], and accordingly, have found a number of clinical applications, including as inlays/onlays, single crown/bridges, implant abutments, orthodontic brackets, and fixed-partial dentures [3,4].

Zirconia, however, comes with a drawback. The absence of a silica glass component and the homogenous, densely packed structure of zirconia crystals make etching and silanization (and therefore durable bonding) difficult $[5,6]$. Strong and reliable bonding with ceramic restorations relies on chemical bonding and micro-mechanical interlocking $[7,8]$. Chemical bonding is achieved using a functional monomer, 10-methacryloyloxydecyl dihydrogen phosphate (MDP) [9-11]. According to 
Noriyuki et al. [12], the chemical bonds between 10-MDP and zirconia are the result of ionic and hydrogen bonding. Other research has shown that the use of a primer and resin cement containing 10-MDP results in a relatively high bond strength in zirconia restorations [13]. However, other studies [5,14] have reported that improved bond strength to zirconia treated with MDP-containing primers cannot be maintained for a long time due to the acceleration of hydrolysis at the bonding site.

Several studies have been undertaken to examine the methods and effects of increased micromechanical interlocking between zirconia restoration and luting resin cement [15-17]. The conventional method of increasing the micromechanical retention by airborne-particle abrasion (sandblasting) with $\mathrm{Al}_{2} \mathrm{O}_{3}$ or $\mathrm{SiO}_{2}$ powder not only weakens the zirconia restoration by causing unfavorable surface modifications such as micropores (which can act as crack initiators), but also induce the zirconia crystals to undergo an unfavorable phase transformation from tetragonal to monoclinic [18-20]. Consequently, this method seems poorly suited for establishing reliable zirconia/resin cement micromechanical interlocking.

Alternative means of modifying the surface of zirconia restorations to increase its micromechanical interlocking with luting resin cement have been proposed in the literature, including selective infiltration etching (SIE) [21], high-temperature chemical etching [22], and hydrofluoric acid (HF) etching [23]. Of these, HF etching has received the most attention. Until recently, the HF etching of zirconia was generally thought to be impossible. However, recent studies have shown that etching is, in fact, possible with adjustments to the various application conditions, including concentration, immersion time, and temperature $[23,24]$. As most studies on HF etching have been conducted at room temperature, this study was designed to investigate changes in the bond strength between resin cement and tetragonal zirconia polycrystalline stabilized with 3 to $8 \mathrm{~mol} \%$ yttrium oxide (Y-TZP) when the HF solution was raised to $70-80^{\circ} \mathrm{C}$. This study also observed the topographical changes of Y-TZP surfaces etched with the HF solution under various concentrations and temperature conditions.

The two null hypotheses of this study were that changes to the concentration and temperature of hydrofluoric acid neither (1) increased the bonding strength between zirconia and resin cement, nor (2) caused topographical changes on the Y-TZP surface.

\section{Materials and Methods}

The materials used in this study are listed in Table 1.

Table 1. Materials used, manufacturers and major components.

\begin{tabular}{ccc}
\hline Material/Trade Name & Manufacturer & Main Components \\
\hline $\begin{array}{c}\text { Hydrofluoric acid (HF) } \\
\text { /Hydrofluoric acid }\end{array}$ & $\begin{array}{c}\text { Merck KGaA, } \\
\text { Darmstadt, } \\
\text { Germany }\end{array}$ & $48 \mathrm{wt} \%$ in $\mathrm{H}_{2} \mathrm{O}$ \\
$\begin{array}{c}\text { Zirconia Primer } \\
\text { /Z-prime Plus }\end{array}$ & $\begin{array}{c}\text { Bisco Inc., } \\
\text { Schaumbrug, IL, } \\
\text { USA }\end{array}$ & BPDM, HEMA, ethanol \\
MDP-containing resin cement & $\begin{array}{c}\text { GC Corp., } \\
\text { Tokyo, } \\
\text { Japan }\end{array}$ & $\begin{array}{c}\text { Paste A: Fluoro-alumino-silicate glass, UDMA, } \\
\text { dimethacrylate, Silicon dioxide } \\
\text { Paste B: Phosphoric acid ester monomer (MDP), } \\
\text { silicon dioxide, UDMA, dimethacrylate }\end{array}$ \\
\hline
\end{tabular}

Abbreviation: BPDM (biphenyl dimethacrylate), HEMA (hydroxyethyl methacrylate), UDMA (urethane dimethacrylate), MDP (10-methacryloyloxydecyl dihydrogen phosphate).

Ninety sintered Y-TZP specimens (NexxZr T, Sagemax, Federal Way, WA, USA) and resin blocks were prepared for the shear bond strength (SBS) test. Y-TZP specimens in the form of standard cylinders (12 $\mathrm{mm}$ in diameter, $8 \mathrm{~mm}$ in height) were prepared by CAD/CAM (Shanghai Machine Tool Ltd., Shanghai, Chaina) milling and sintering. The resin blocks were prepared by incremental packing (Unifil Loflo, GC Inc., Tokyo, Japan) into a cylindrical Teflon mold (3 mm in diameter, $10 \mathrm{~mm}$ in height) and then light-curing each increment for $20 \mathrm{~s}$. 
Non-etched sintered Y-TZP specimens $(n=10)$ were used as the control. Other experimental groups were etched with varied concentrations of HF under varied temperature conditions (Figure 1). HF solutions (of 5\%,10\%, 20\%, and 40\% concentration) were experimentally prepared using $48 \% \mathrm{HF}$ solution (MKBH5499V, Sigma-Aldrich Co., St. Louis, MO, USA), distilled water, and an electronic scale. Under two different temperature conditions (room temperature, $70-80{ }^{\circ} \mathrm{C}$ ), ten Y-TZP specimens for each experimental group were etched with 5\%,10\%, 20\%, and 40\% HF solutions for 10 min in a plastic box. The specimens were then 'hot etched'; a water bath was used to raise the temperature of the HF solution to $70-80^{\circ} \mathrm{C}$. After etching, the Y-TZP specimens were ultrasonically cleaned in distilled water for $10 \mathrm{~min}$ and then gently air-dried.

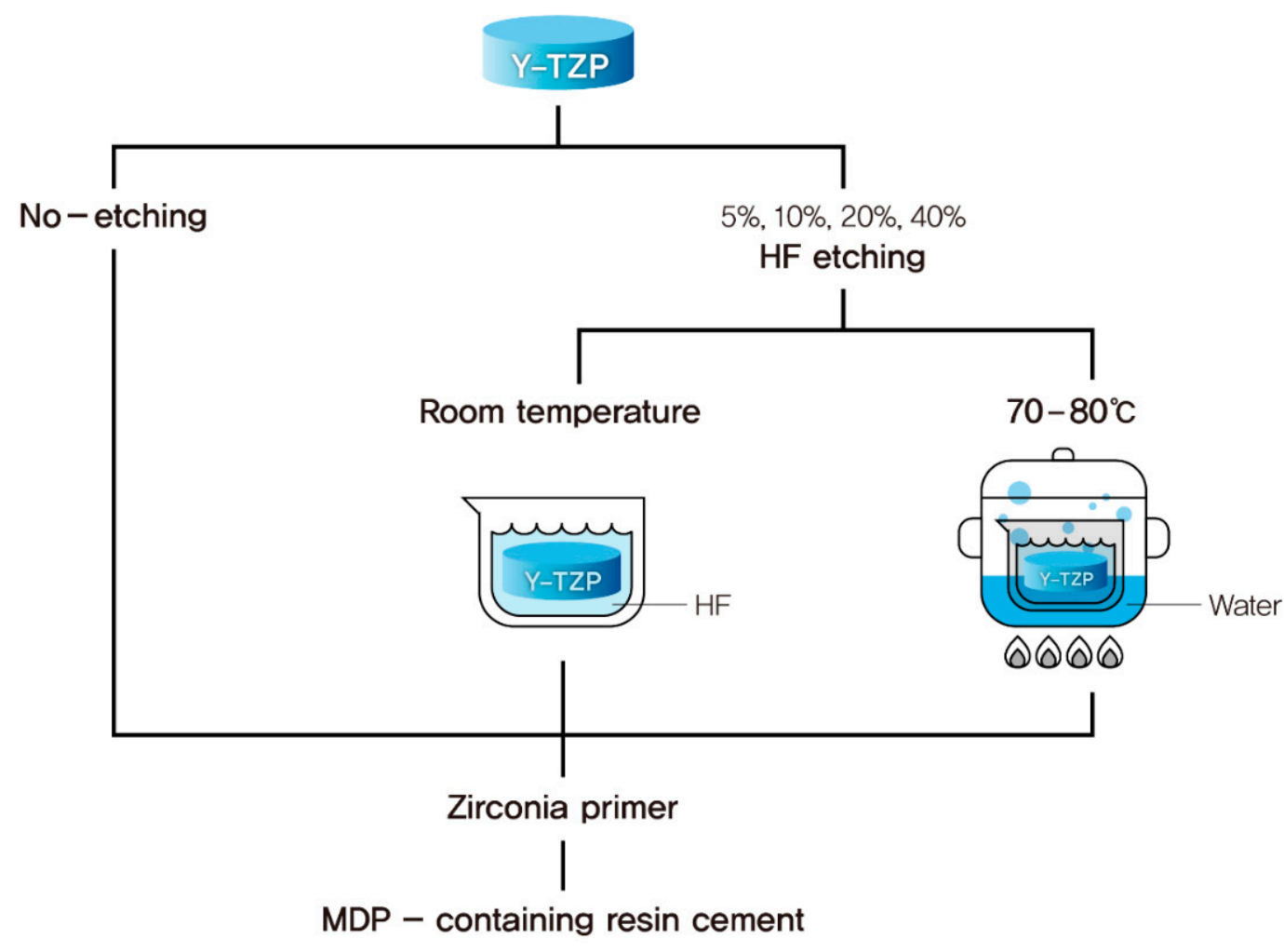

Figure 1. Schematic representation of the pretreatment procedures undergone by the zirconium oxide (Y-TZP) specimen following different hydrofluoric acid (HF) etching conditions.

Zirconia primer (Z-prime plus, Bisco Inc., Schaumburg, IL, USA) was applied to the surface of all groups of Y-TZP for $10 \mathrm{~s}$, after which all groups were air-dried for $15 \mathrm{~s}$. Resin cement containing MDP (G-CEM LinkAce, GC Inc.) was then applied to all groups. Under a constant load of $5 \mathrm{~N}$, light curing was performed for $3 \mathrm{~s}$ for tack curing, and the excess resin cement was removed using hand instruments. While maintaining the constant load, all surfaces were light-cured for $20 \mathrm{~s}$. The specimens that underwent luting procedures were stored in distilled water at $36^{\circ} \mathrm{C}$ for $24 \mathrm{~h}$ before the SBS test.

Specimens were then mounted in the jig of a universal testing machine (Model 5543, Instron, Canton, MA, USA) and shear force (using a chisel-shaped metal rod at a constant crosshead speed of $0.5 \mathrm{~mm} / \mathrm{min}$ ) was applied to the specimen until failure. Load was measured by recording the force at which the failure occurred, and the SBS was calculated using the following formula: SBS $(\mathrm{MPa})=$ load $(\mathrm{N}) /$ area $\left(\mathrm{mm}^{2}\right)$.

One Y-TZP disk specimen from each HF etch group was rinsed with 95\% ethanol, air dried, mounted on a metal stub, and coated with a gold-palladium alloy using sputter coating technology (Ion sputter E-1030, Hitachi, Tokyo, Japan). Subsequently, the structural change of each HF-etched 
Y-TZP surface was observed at 10,000 times magnification with a scanning electron microscope (SEM) (SU8230, Hitachi, Tokyo, Japan).

One Y-TZP disk specimen from each HF etch group was used for the morphological change and surface roughness analysis using atomic force microscopy (AFM) (Multimode-8, Bruker, Santa Barbara, CA, USA). To analyze the surface roughness of each group of HF etched Y-TZP specimens, a silicon AFM probe $(\mathrm{k}=42 \mathrm{~N} / \mathrm{m}, \mathrm{f}=320 \mathrm{kHz}$, NCHR, Nanoworld, Neuchâtel, Switzerland) was used to perform in tapping mode with a scan size of $5 \times 5 \mu \mathrm{m}$. Five measurements were performed for each specimen and the average was determined as the mean Ra value.

Statistical analysis was performed using statistical software (SPSS Version 18.0, SPSS Inc., Chicago, IL, USA). The mean and standard deviation (SD) of SBS $(n=10)$ and surface roughness Ra values were statistically analyzed using two-way analysis of variance (ANOVA), and significant differences between the groups were determined using the pairwise multiple comparison procedure (Tukey's test). $p$-values less than 0.05 were considered statistically significant in all tests.

\section{Results}

The mean SBS values with standard deviations are presented in Figure 2.

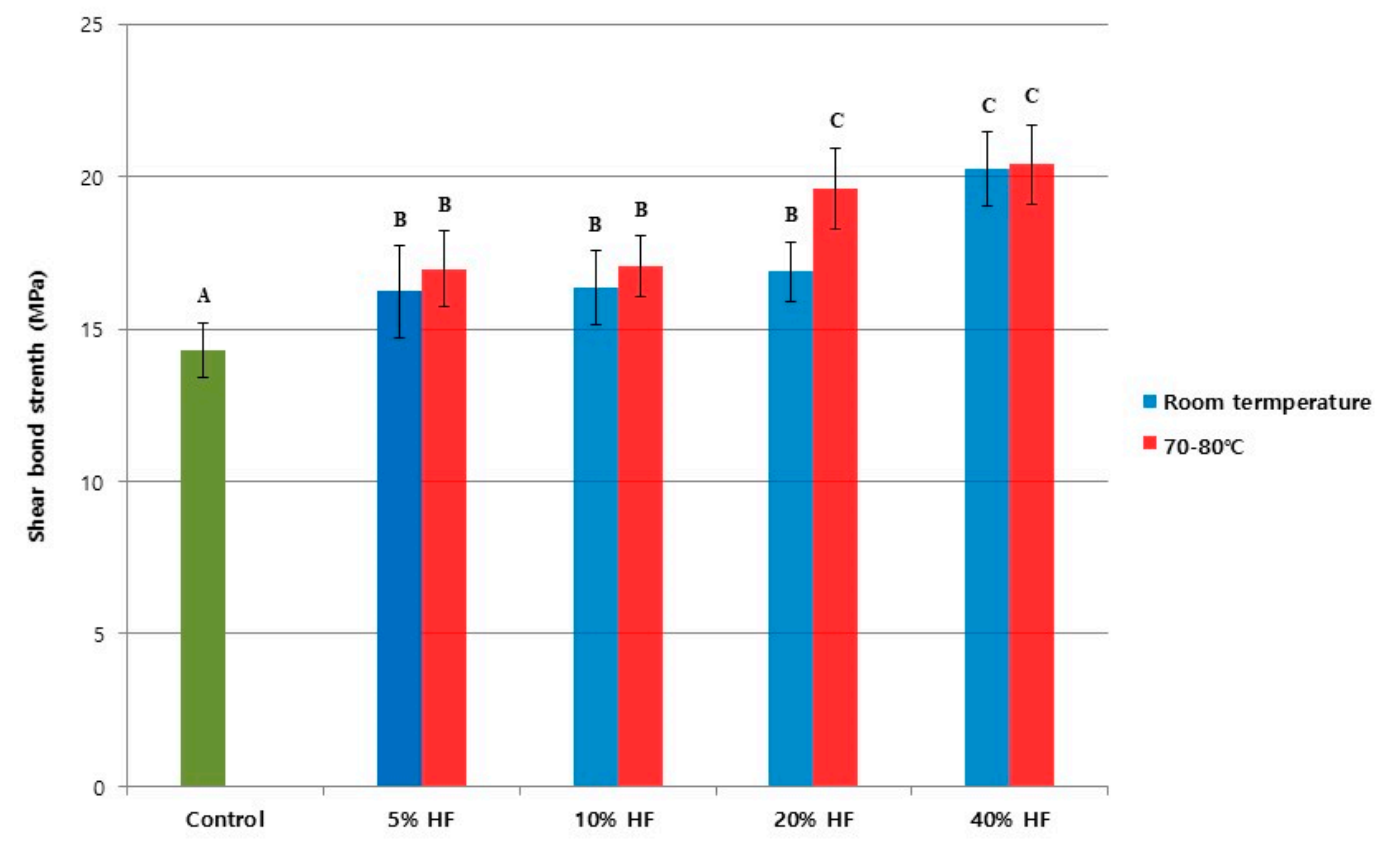

Figure 2. Shear bond strengths (MPa) of the control group that did not etch the surface and the experimental groups that did etch the Y-TZP surface with hydrofluoric acid under various conditions. Identical capital letters indicate that there are no statistically significant differences $(p>0.05)$.

All HF etching groups showed significantly higher SBS values when compared to the control group $(p<0.05)$. Two-way ANOVA showed that both concentrations of the HF solution and the etching temperature had a significant effect on SBS $(p<0.05)$. Interactions between the various concentrations of HF solutions and the etching temperature conditions were also statistically significant $(p<0.05)$.

At room temperature, the highest SBS value was measured in the $40 \%$ HF etching group, representing a significant difference compared to the other groups $(p<0.05)$. No statistically significant differences were found between the $5 \%, 10 \%$ and $20 \%$ HF etching groups $(p>0.05)$, although these HF etching groups did show higher SBS values than the control $(p<0.05)$. Consistent with the test undertaken under room temperature conditions, at $70-80^{\circ} \mathrm{C}$, the $40 \% \mathrm{HF}$ etching group had the highest SBS value. Here, however, there were no significant difference from the $20 \%$ HF etching group $(p>0.05)$ under these temperature conditions. Overall, the $20 \% \mathrm{HF}$ etching group at $70-80{ }^{\circ} \mathrm{C}$, as well as the 
$40 \% \mathrm{HF}$ etching groups at both room temperature and $70-80{ }^{\circ} \mathrm{C}$, were significantly different from the other groups $(p<0.05)$.

Representative SEM images of HF etched Y-TZP specimens under different conditions are shown in Figure 3. SEM observation revealed that the $40 \%$ HF etching groups from both the room temperature and the $70-80^{\circ} \mathrm{C}$ tests showed a completely different morphology compared to the control group. The dissolution of the Y-TZP surface grain structure by the HF solution increased with the concentration and application temperature, resulting in high surface roughness and irregularities. However, among the low concentration $(5 \%, 10 \%)$ HF etching groups, the solution was not effective for etching Y-TZP surfaces regardless of temperature.

Representative AFM images of HF etched Y-TZP specimens under different conditions are shown in Figures 4 and 5. The AFM images also revealed that the HF solution could etch the Y-TZP surface. The mean Ra values increased along with temperature in all HF etching groups. The $5 \%$ HF etching group had the least increase in the mean Ra value, the $10 \%$ and $20 \%$ HF etching groups showed similar increases, and the $40 \% \mathrm{HF}$ etching group had the largest increase. The $40 \% \mathrm{HF}$ etching group at room temperature showed a significantly higher Ra value, than that which was apparent in the other HF etching groups $(p<0.05)$. There was no significant difference in the mean Ra values between the $5 \%, 10 \%$, and $20 \% \mathrm{HF}$ etching groups at room temperature $(p>0.05)$. The $40 \% \mathrm{HF}$ etching group also displayed a significantly higher mean Ra value than the other HF etching groups $(p<0.05)$ at temperatures of $70-80{ }^{\circ} \mathrm{C}$. In these tests, unlike the room temperature tests, the $10 \%$ and $20 \% \mathrm{HF}$ etching groups showed significantly higher mean Ra values than the $5 \%$ HF etching group $(p<0.05)$. 

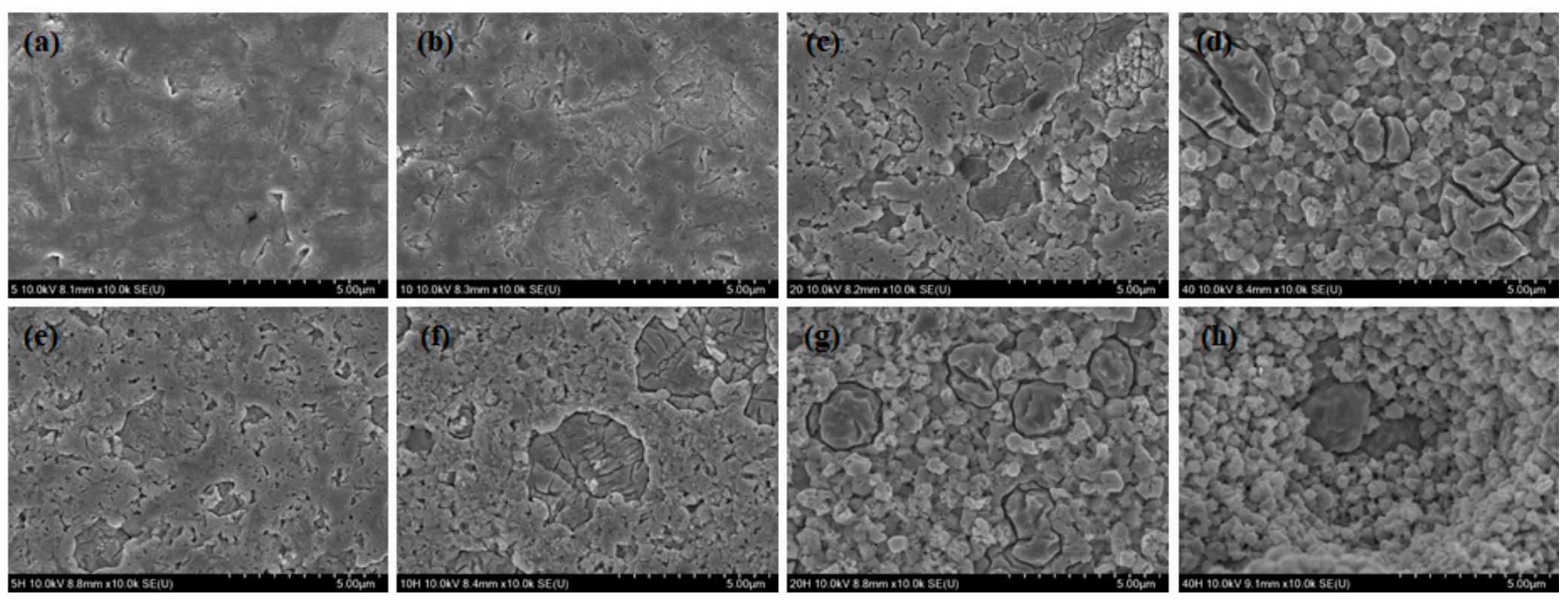

Figure 3. SEM images of the Y-TZP surface etched with hydrofluoric acid (HF) under various conditions: (a) room temperature: $5 \% \mathrm{HF}$; (b) room temperature, $10 \%$ $\mathrm{HF}$; (c) room temperature, $20 \% \mathrm{HF}$; (d) room temperature, $40 \% \mathrm{HF}$; (e) $70-80{ }^{\circ} \mathrm{C}, 5 \% \mathrm{HF}$; (f) $70-80{ }^{\circ} \mathrm{C}, 10 \% \mathrm{HF}$; (g) $70-80{ }^{\circ} \mathrm{C}, 20 \% \mathrm{HF}$; (h) $70-80{ }^{\circ} \mathrm{C}, 40 \% \mathrm{HF}$. 

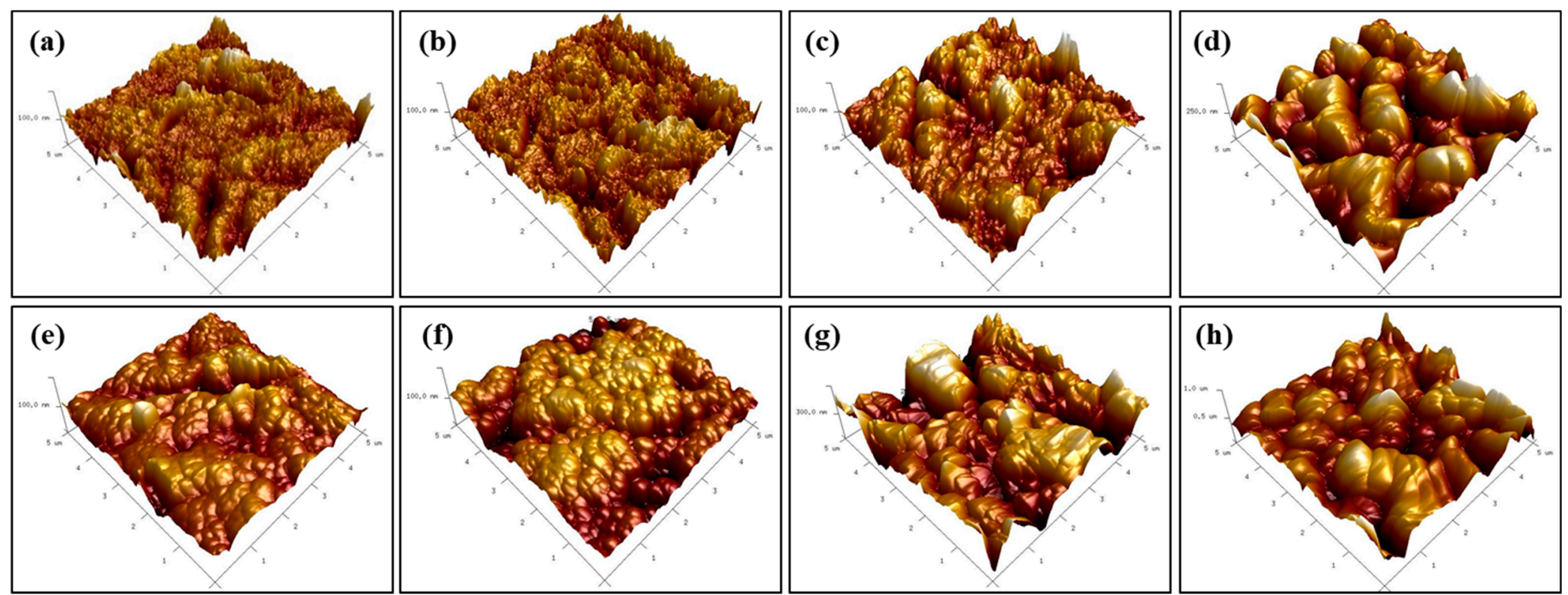

Figure 4. AFM images of the Y-TZP surface etched with hydrofluoric acid (HF) under various conditions: (a) room temperature, $5 \% \mathrm{HF}$; (b) room temperature, $10 \% \mathrm{HF}$; (c) room temperature, $20 \% \mathrm{HF}$; (d) room temperature, $40 \% \mathrm{HF}$; (e) $70-80{ }^{\circ} \mathrm{C}, 5 \% \mathrm{HF}$; (f) $70-80{ }^{\circ} \mathrm{C}, 10 \% \mathrm{HF}$; (g) $70-80{ }^{\circ} \mathrm{C}, 20 \% \mathrm{HF}$; (h) $70-80{ }^{\circ} \mathrm{C}, 40 \% \mathrm{HF}$. 


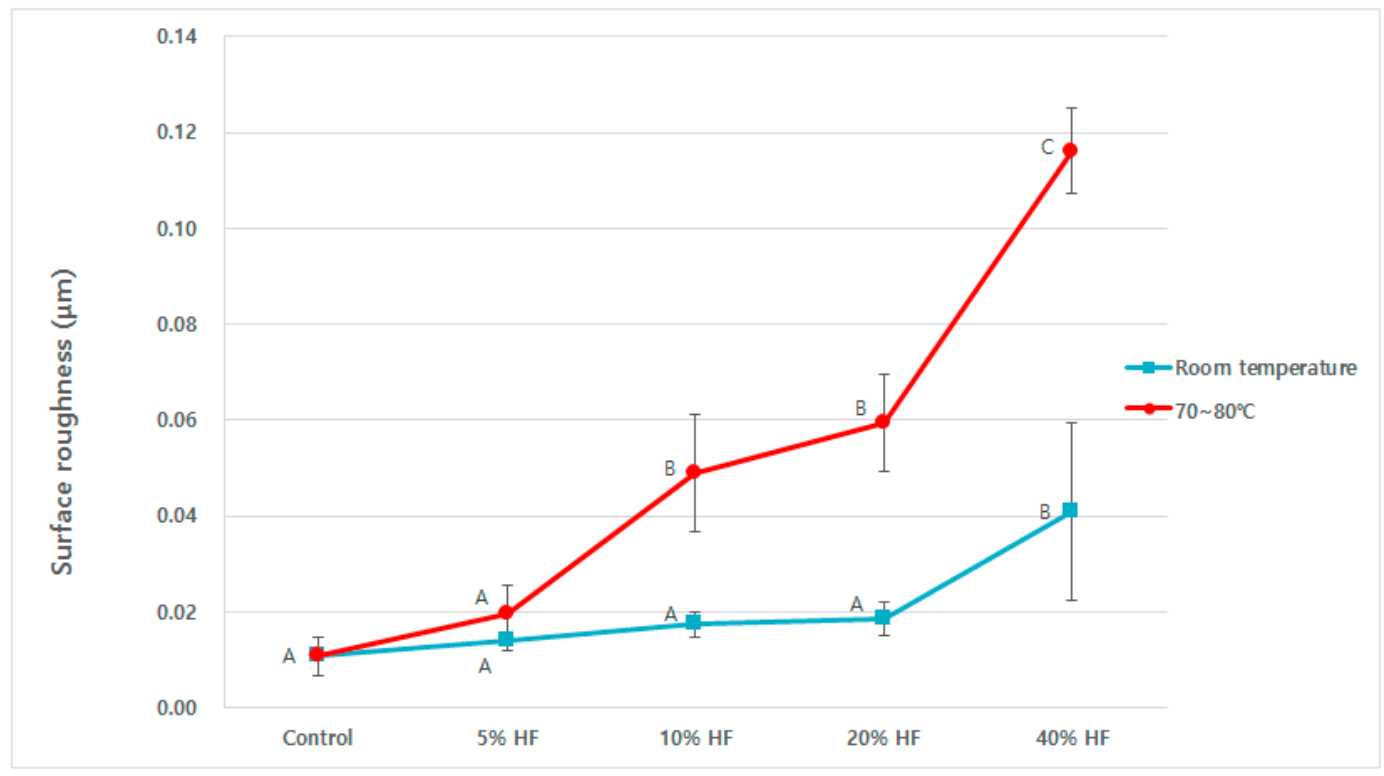

Figure 5. Mean surface roughness ( $\mathrm{Ra}$ ) of Y-TZP etched with hydrofluoric acid depending on the concentration and temperature conditions. Identical uppercase letters indicate no statistically significant differences $(p>0.05)$.

\section{Discussions}

Hydrofluoric acid is an inorganic compound consisting of hydrogen fluoride (HF) in water. HF is used in industrial processes such as glass etching, metal cleaning, and silicon wafer etching, and as laboratory reagents $[25,26]$. In dentistry, a concentration of $4-10 \% \mathrm{HF}$ is used as a magnetic/ceramic surface conditioner [27]. HF has a distinct ability to dissolve the glass phase, leaving behind a crystalline phase of porcelain/ceramic and with a rough surface. However, it is a well-known limitation of HF that it does not affect zirconia due to its high crystallinity and lack of glass phase $[5,6]$.

This study has shown that conventional knowledge is only partially correct. In fact, under the right conditions, HF can etch zirconia. SEM and AFM images of HF etched Y-TZP specimens revealed that an HF solution partially etched the Y-TZP superficial grain structure (in), leading to the formation of irregular grooves (increasing the inter-grain size) and nano-sized porosity (reducing the grain size). Etching can also occur inside the grains of the Y-TZP specimens, as atoms around the crystal boundary can dissolve faster than atoms inside the crystal [23,28].

There are few studies on the Y-TZP etching mechanism by HF. Flamant et al. [29] that attempt to theoretically explain the dissolution of $\mathrm{ZrO}_{2}$ in $\mathrm{HF}$, based on the Pourbaix speciation diagrams. According to Lowalekar et al. [30], element $\mathrm{F}$ in a $40 \%$ HF solution dissolved zirconium oxide and yttrium oxide to form fluoride, oxide and hydroxide complexes.

In this experiment, not only at room temperature, but also how efficiently the Y-TZP surface is etched by hydrofluoric acid at $70-80^{\circ} \mathrm{C}$, was investigated. This is because it was thought that even at the same concentration, increasing the temperature would increase the surface area etched by hydrofluoric acid, resulting in an increase in micromechanical bonds. However, since hydrofluoric acid has a low boiling point, it can be easily vaporized and can be very harmful to the human body. In addition, in this experiment, since a water bath was used, the degree of etching by hydrofluoric acid was studied at a temperature of $70-80^{\circ} \mathrm{C}$ lower than the boiling point of water, $100{ }^{\circ} \mathrm{C}$.

Our study showed that a surface change and a significant increase in the mean Ra value were observed from the $10 \% \mathrm{HF}$ etched group at $70-80{ }^{\circ} \mathrm{C}$, but not the group etched at room temperature. It was also found that as the concentration of the HF solution increased, the higher the average Ra value rose as the temperature increased. As both the temperature and concentration increased, the HF solution became increasingly molecularly reactive, making it possible to etch the Y-TZP specimen 
surface much faster and more effectively. Yu et al. [31] reportedly achieved similar results by applying HF-based zirconia etching solution at high temperature. Casucci et al. [22] also reported that when etching the zirconia surface with a hot acidic solution $\left(100^{\circ} \mathrm{C}\right)$, the inter-particle spacing and surface roughness increased significantly. This technique can only be helpful in increasing the etching efficiency for Y-TZP up to a point; when the HF solution is heated past its boiling point, it inevitably produces extremely dangerous and potentially lethal HF vapor [32].

There is a controversy concerning whether HF etching pretreatment for Y-TZP improves the bond strength. Some studies have reported that HF etching for Y-TZP does not improve bond strength $[23,24,33]$. According to their research results, nano-sized porosity and irregular pattern structures were formed on the HF-etched Y-TZP surface, but the viscosity of the resin cement was not low enough, suggesting that micromechanical interlocking was not properly formed. They also suggested that HF etching induced the phase transformation from tetragonal to monoclinic structure of Y-TZP due to the low-temperature degradation associated with humid conditions [34,35]. Moreover, they concluded that the phase transformation caused by this degradation did not improve the bonding strength by reducing the physical properties of Y-TZP. Lee et al. [36], however, reported that the phase transformation from tetragonal to monoclinic structure was observed in all HF etching groups, but as the monoclinic structure of the HF etching group was smaller than that of the airborne-particle abrasion group, the HF etching itself did not induce the sufficient phase transformation to the extent that it adversely affected the bond strength to Y-TZP.

The present study showed that the HF etching pretreatment improved the bond strength to Y-TZP compared to the control. In our study, the low-viscosity resin cement penetrated into the irregularly patterned fine-sized porous structure formed after HF etching. The higher the mean Ra value, the stronger the micromechanical linkage could be formed, so the higher the bond strength was measured at a higher mean Ra value.

There was no difference in adhesive strength between the $40 \%$ HF etching groups, though the mean Ra value was higher at $70-80{ }^{\circ} \mathrm{C}$ than at room temperature. The SEM image of the $40 \% \mathrm{HF}$ etching group at $70-80{ }^{\circ} \mathrm{C}$ showed that the $\mathrm{Y}$-TZP surface was not uniformly etched, and that a part of the sample was much more deeply recessed compared to the nearby parts. Non-homogeneous etching like this would have made it difficult to achieve strong micromechanical interlocking and is the reason that the bond strength did not increase despite the growth in the mean Ra value. These results indicate that there is no advantage to dangerously increasing the temperature of hydrofluoric acid when etching Y-TZP with a 40\% HF solution.

Based on these results, both the null hypotheses initially proposed were rejected. Further studies are needed to achieve a more detailed understanding of the mechanism by which HF etches the Y-TZP surface and the most suitable etching conditions (concentration, temperature, and immersion time) that will improve the bonding strength of the Y-TZP surface.

\section{Conclusions}

Within the limits of this study, pretreatment with $20 \% \mathrm{HF}$ solution at $70-80{ }^{\circ} \mathrm{C}$ or $40 \% \mathrm{HF}$ solution at room temperature and $70-80^{\circ} \mathrm{C}$ can effectively acid-etch the Y-TZP surface, resulting in high surface roughness and irregularities. However, accounting for the concentration and temperature conditions of the HF solution, $40 \%$ HF solution at room temperature is recommended to improve the adhesion between the resin cement and Y-TZP.

Author Contributions: Conceptualization, M.-J.L. and K.-W.L.; methodology, H.-E.K. and M.-J.L.; validation, M.-J.L., M.-K.Y. and K.-W.L.; investigation, H.-E.K. and M.-J.L.; writing-original draft preparation, H.-E.K. and M.-J.L.; writing-review and editing, M.-K.Y. and K.-W.L.; supervision, M.-K.Y. and K.-W.L. All authors have read and agreed to the published version of the manuscript.

Funding: This research received no external funding.

Acknowledgments: This research was supported by Biomedical Research Institute, Jeonbuk National University Hospital, grant number CUH2020-0036. 
Conflicts of Interest: The authors declare no conflict of interest.

\section{References}

1. Denry, I.; Kelly, J.R. State of the art of zirconia for dental applications. Dent. Mater. 2008, 24, $299-307$. [CrossRef] [PubMed]

2. Ozcan, M.; Vallittu, P.K. Effect of surface conditioning methods on the bond strength of luting cement to ceramics. Dent. Mater. 2003, 19, 725-731. [CrossRef]

3. Manicon, P.F.; Iommetti, P.R.; Raffaelli, L. An overview of zirconia ceramics: Basic properties and clinical applications. J. Dent. 2007, 35, 819-826. [CrossRef] [PubMed]

4. Beuer, F.; Schweiger, J.; Edelhoff, D. Digital dentistry: An overview of recent developments for CAD/CAM generated restorations. Br. Dent. J. 2008, 204, 505-511. [CrossRef] [PubMed]

5. Blatz, M.B.; Sadan, A.; Kern, M. Resin-ceramic bonding: A review of the literature. J. Prosthet. Dent. 2003, 89, 268-274. [CrossRef] [PubMed]

6. Derand, T.; Molin, M.; Kvam, K. Bond strength of composite luting cement to zirconia ceramic surfaces. Dent. Mater. 2005, 21, 1158-1162. [CrossRef] [PubMed]

7. Thompson, J.Y.; Stoner, B.R.; Piascik, J.R.; Smith, R. Adhesion/cementation to zirconia and other non-silicate ceramics: Where are we now? Dent. Mater. 2011, 27, 71-82. [CrossRef]

8. Burke, F.J.; Fleming, G.J.; Nathanson, D.; Marquis, P.M. Are adhesive technologies needed to support ceramics? An assessment of the current evidence. J. Adhes Dent. 2002, 4, 7-22.

9. Yoshida, K.; Yamashita, M.; Atsuta, M. Zirconate coupling agent for bonding resin luting cement to pure zirconium. Am. J. Dent. 2004, 17, 249-252.

10. Yoshida, K.; Tsuo, Y.; Atsuta, M. Bonding of dual-cured resin cement to zirconia ceramic using phosphate acid ester monomer and zirconate coupler. J. Biomed. Mater. Res. B Appl. Biomater. 2006, 77, 28-33. [CrossRef]

11. Kitayama, S.; Nikaido, T.; Takahashi, R.; Zhu, L.; Ikeda, M.; Foxton, R.M.; Sadr, A.; Tagami, J. Effect of primer treatment on bonding of resin cements to zirconia ceramic. Dent. Mater. 2010, 26, 426-432. [CrossRef] [PubMed]

12. Nagaoka, N.; Yoshihara, K.; Feitosa, V.P.; Tamada, Y.; Irie, M.; Yoshida, Y.; Van Meerbeek, B.; Hayakawa, S. Chemical interaction mechanism of 10-MDP with zirconia. Sci. Rep. 2017, 7, 17. [CrossRef]

13. Lim, M.J.; Yu, M.K.; Lee, K.W. The effect of continuous application of MDP-containing primer and luting resin cement on bond strength to tribochemical silica-coated Y-TZP. Restor. Dent. Endod. 2018, 43, e19. [CrossRef] [PubMed]

14. Attia, A. Bond strength of three luting agents to zirconia ceramic-influence of surface treatment and thermocycling. J. Appl. Oral Sci. 2011, 19, 388-395. [CrossRef] [PubMed]

15. Tanaka, R.; Fujishima, A.; Shibata, Y.; Manabe, A.; Miyazaki, T. Cooperation of phosphate monomer and silica modification on zirconia. J. Dent. Res. 2008, 87, 666-670. [CrossRef] [PubMed]

16. Rüttermann, S.; Fries, L.; Raab, W.H.; Janda, R. The effect of different bonding techniques on ceramic/resin shear bond strength. J. Adhes Dent. 2008, 10, 197-203. [PubMed]

17. Piwowarczyk, A.; Lauer, H.C.; Sorensen, J.A. The shear bond strength between luting cements and zirconia ceramics after two pre-treatments. Oper. Dent. 2005, 30, 382-388.

18. Cavalcanti, A.N.; Foxton, R.M.; Watson, T.F.; Oliveira, M.T.; Giannini, M.; Marchi, G.M. Bond strength of resin cements to a zirconia ceramic with different surface treatments. Oper. Dent. 2009, 34, 280-287. [CrossRef]

19. Guazzato, M.; Albakry, M.; Quach, L.; Swain, M.V. Influence of surface and heat treatments on the flexural strength of a glass-infiltrated alumina/zirconia-reinforced dental ceramic. Dent. Mater. 2005, 21, 454-463. [CrossRef]

20. Passos, S.P.; Linke, B.; Major, P.W.; Nychka, J.A. The effect of air-abrasion and heat treatment on the fracture behavior of Y-TZP. Dent. Mater. 2015, 31, 1011-1021. [CrossRef]

21. Aboushelib, M.N.; Kleverlaan, C.J.; Feilzer, A.J. Selective infiltration-etching technique for a strong and durable bond of resin cements to zirconia-based materials. J. Prosthet. Dent. 2007, 98, 379-388. [CrossRef]

22. Casucci, A.; Osorio, E.; Osorio, R.; Monticelli, F.; Toledano, M.; Mazzitelli, C.; Ferrari, M. Influence of different surface treatments on surface zirconia frameworks. J. Dent. 2009, 37, 891-897. [CrossRef]

23. Sriamporn, T.; Thamrongananskul, N.; Busabok, C.; Poolthong, S.; Uo, M.; Tagami, J. Dental zirconia can be etched by hydrofluoric acid. Dent. Mater. J. 2014, 33, 79-85. [CrossRef] [PubMed] 
24. Chaiyabutr, Y.; McGowan, S.; Phillips, K.M.; Kois, J.C.; Giordano, R.A. The effect of hydrofluoric acid surface treatment and bond strength of a zirconiaveneering ceramic. J. Prosthet. Dent. 2008, 100, 194-202. [CrossRef]

25. Genuino, H.C.; Opembe, N.N.; Njagi, E.C.; McClain, S.; Suib, S.L. A review of hydrofluoric acid and its use in the car wash industry. J. Ind. Eng. Chem. 2012, 18, 1529-1539. [CrossRef]

26. Ozcan, M.; Allahbeickaraghi, A.; Dundar, M. Possible hazardous effects of hydrofluoric acid and recommendations for treatment approach: A review. Clin. Oral Investig. 2012, 16, 15-23. [CrossRef] [PubMed]

27. Alex, G. Preparing porcelain surfaces for optimal bonding. Compend. Contin. Educ. Dent. 2008, 29, 324-335.

28. Callister, W.D., Jr.; Rethwisch, D.G. Materials Science and Engineering: An Introduction, 8th ed.; John Wiley SonsInc: New York, NY, USA, 2010.

29. Flamant, Q.; Marro, F.G.; Rovira, J.J.R.; Anglada, M. Hydrofluoric acid etching of dental zirconia. Part 1: Etching mechanism and surface characterization. J. Eur Ceram. Soc. 2016, 36, 1211-1234. [CrossRef]

30. Lowalekar, V.; Raghavan, S. Etching of zirconium oxide, hafnium oxide, and hafnium silicates in dilute hydrofluoric acid solutions. J. Mater. Res. 2004, 19, 1149-1156. [CrossRef]

31. Yu, M.K.; Lim, M.J.; Na, N.R.; Lee, K.W. Effect of hydrofluoric acid-based etchant at an elevated temperature on the bond strength and surface topography of Y-TZP ceramics. Restor. Dent. Endod. 2019, 45, e6. [CrossRef]

32. Hawkinson, T.E.; Korpela, D.B. Chemical hazards in semiconductor operations. In Semiconductor Safety Handbook: Safety and Health in the Semiconductor Industry; William Andrew: New York, NY, USA, 1997.

33. Smielak, B.; Klimek, L. Effect of hydrofluoric acid concentration and etching duration on select surface roughness parameters for zirconia. J. Prosthet. Dent. 2015, 113, 596-602. [CrossRef]

34. Lughi, V.; Sergo, V. Low temperature degradation-Aging-of zirconia: A critical review of the relevant aspects in dentistry. Dent. Mater. 2010, 26, 807-820. [CrossRef] [PubMed]

35. Chevalier, J.; Gremillart, L.; Deville, S. Low-Temperature Degradation of Zirconia and Implications for Biomedical Implants. Annu. Rev. Mater. Res. 2007, 37, 1-32. [CrossRef]

36. Lee, M.H.; Son, J.S.; Kim, K.H.; Kwon, T.Y. Improved resin-zirconia bonding by room temperature hydrofluoric acid etching. Materials 2015, 8, 8508-8566. [CrossRef] [PubMed]

Publisher's Note: MDPI stays neutral with regard to jurisdictional claims in published maps and institutional affiliations.

(C) 2020 by the authors. Licensee MDPI, Basel, Switzerland. This article is an open access article distributed under the terms and conditions of the Creative Commons Attribution (CC BY) license (http://creativecommons.org/licenses/by/4.0/). 